\title{
Drowning in Pretoria, South Africa: A 10-year review
}

\author{
N.K. Morris, L. du Toit-Prinsloo and G. Saayman
}

Affiliation and addresses of the authors:

N.K. Morris:

Lecturer; University of Pretoria; Department of Forensic Medicine, Private Bag X323, Arcadia, 0007 SOUTH AFRICA

BSc, BSc(hons), MSc(medical criminalistics)

L. du Toit-Prinsloo:

Specialist / Lecturer; University of Pretoria; Department of Forensic Medicine, Private Bag X323,

Arcadia, 0007 SOUTH AFRICA

MBChB, DipForMed(SA)Path, FCForPath(SA), MMed(Path)(Forens)

G. Saayman:

Chief specialist / Head of Department; University of Pretoria; Department of Forensic Medicine, Private Bag X323, Arcadia, 0007 SOUTH AFRICA

MBChB, MMed(MedForens), FCForPath(SA)

E-mail address, telephone and fax numbers of the corresponding author:

L. du Toit-Prinsloo

Email: $\quad$ lorraine.dutoit@up.ac.za

Telephone numbers: $\quad$ +27 (0)123235298 (work)

+27 (0)834064056 (mobile)

Fax number: $\quad$ + $\quad$ +27(0)123230921

\section{Conflict of interest disclosure}

There is no conflict of interest.

\section{Funding}

No funding was obtained for this study

\section{Highlights:}

- Most drowning deaths in Pretoria are amongst children who die in swimming pools.

- Especially in children, these are preventable deaths and renewed efforts should be made at improving public awareness and implementing preventive measures.

- Alcohol is present amongst adults who drown. 
- A thorough investigation of immersion-related deaths should be done as drowning is not an easy diagnosis at autopsy alone.

\begin{abstract}
:
Drowning is classified as the 3rd leading cause of accidental deaths worldwide and deemed to be a preventable cause of death. Bodies retrieved from a water medium pose several challenges to the forensic pathologist, with the diagnosis of drowning being primarily one of exclusion. The aim of this study was to do a retrospective descriptive case audit of bodies retrieved from water and immersion related deaths which were investigated at the Pretoria Medico-Legal Laboratory (PMLL) over a 10 year period (January 2002 through December 2011). A total of 346 cases were identified for inclusion into this study. In 6\% (20) of these cases, the death was not related to drowning; in 14\% (48) no clear cause of death could be ascertained and in 278 cases $(80 \%)$ the cause of death was considered to have been due to drowning. Infants (under 1 year of age) constituted 41 (15\%) of the cases; toddlers (aged 1-2 years) comprised 52 (19\%) cases; children (aged 2-13 years) 49 (18\%) cases; adolescents (aged 13-18 years) comprised 10 (3\%) cases; adults (above 18 years) made up 126 (45\%) of the cases. The majority of the drownings occurred in swimming pools [125 cases (38\%)]. In infants, 23 (56\%) of drownings occurred in swimming pools followed by buckets [7 cases (17\%)]. Sixty-nine per cent of toddler drownings (36 cases) occurred in swimming pools. In the adult population, 40 (32\%) of cases occurred in pools and 35 cases (28\%) in rivers.. Positive blood alcohol results were found in 48 (42\%) out of 113 cases where the test was requested, $40(35 \%)$ of these cases higher than $0.05 \mathrm{~g}$ per $100 \mathrm{ml}$. This study suggests that many drowning deaths in Pretoria may be preventable by introducing greater public awareness of the risks and instituting relatively simple protective measures.
\end{abstract}

\title{
Key words:
}

Bodies retrieved from water; Childhood deaths ; Drowning ; Emhysema aquosum ; Hemolytic staining of the aorta ; Medico-legal investigation of death.

\section{Introduction:}

There are several definitions for drowning in the literature. The World Health Organization (WHO) defines drowning as a "process of experiencing respiratory impairment from submersion/immersion in liquid". ${ }^{1}$ Roll states that "death by drowning is the result of hampering of respiration by obstruction of mouth and nose by a fluid medium (usually water)". ${ }^{2}$ In 2011, Richards asserted that "drowning is responsible for significant preventable morbidity and mortality worldwide, causing more deaths than war". 3 A 2012 UNICEF report classified drowning as the 3rd leading cause of accidental deaths worldwide (with approximately 359000 fatalities) whilst the WHO has reported that the highest rates of drowning worldwide are in Africa (stated to be 10 to 13 times higher than rates documented for the United Kingdom or Germany)., ${ }^{3,4}$

Bodies retrieved from a water medium pose several recognized challenges to the forensic pathologist. In essence, the diagnosis of drowning is one of exclusion. Scenes of death may be difficult to evaluate, whilst decomposition of bodies will add to diagnostic challenges. In the study reported by Ambade et al $31.2 \%$ of bodies retrieved from water were decomposed, as opposed to $1.6 \%$ of all other cases admitted to the mortuary. ${ }^{6,7}$ There are no pathognomonic features to diagnose drowning at autopsy. External signs indicative of immersion include (amongst others) "washer-woman" changes of the skin and a plume of froth at the nostrils. ${ }^{6,8-10}$ Internal examination may also reveal non-specific findings such as pleural effusions and an increase in lung weights. ${ }^{6,8,10}$ Intimal staining of the aorta has recently been reported to be present in $5 \%$ of cases. ${ }^{11}$ Even histological findings in lung sections are regarded as non-specific for drowning. ${ }^{10}$ Although all of these features may be nonspecific, there are clear advantages in conducting a full autopsy in cases of presumed drowning: this enables the pathologist to at least observe and record the presence (or absence) of such non-specific findings which may be associated with drowning or immersion and to identify the presence of underlying natural disease processes (which could have precipitated the event or contributed to the demise of the victim) ${ }^{12}$. In addition, the possible presence and causative association of medication and drugs (such as alcohol) can be investigated. ${ }^{10,12-14}$ 
Drowning is seen as a preventable death, according to the UNICEF report on drowning in children, stating that "Drowning is a significant, preventable cause of death among children in Low and Middle Income Countries. The challenge is to transform this previously unidentified public health issue from a neglected issue to one addressed by national, regional or global level programs." ${ }^{\text {B }}$ Bamber et al reported on 28 infants and children who died over a 16 year period in the region of London, in the UK, from 1995 to $2010 .{ }^{15}$ In this study the majority of children could not be resuscitated, $70 \%$ of drownings occurred at home (in baths or swimming pools) and in $91 \%$ of cases no adult supervision was present at the time of the incident. ${ }^{15}$ Many childhood deaths by drowning could have been prevented by appropriate education and with interventions such as erecting fences around swimming pools. ${ }^{15}$ From China however, Fang et al reported that the majority of childhood drownings from 2001 to 2005 ( $72 \%$ of cases) occurred in natural bodies of water with only $3 \%$ occurring at home. ${ }^{16}$

In South Africa, drowning related fatalities were reported to be the $5^{\text {th }}$ leading cause of accidental deaths in 2010, with 1428 such deaths documented by Statistics South Africa. ${ }^{17}$ Donson et al reported on unintentional drownings in urban South Africa for the period 2001 - 2005 and recorded the drowning rate for Pretoria as being 1.4/100 000 and the drowning rate amongst the 0-4 year old age group as being 6.9/100 000. ${ }^{18}$ Richards indicated that drowning is the second cause of injury mortality in children under 15 years of age. ${ }^{3}$

As relatively little has been reported in the literature on drowning deaths in South Africa, it was the aim of this study to review the institutional records at a large (inland) urban medico-legal mortuary in South Africa, where the body of a deceased presented to us as a death which was probably or possibly due to immersion in a fluid medium. This study may add to our overall understanding of the problem and may help to identify patterns or trends in drowning deaths, especially amongst the pediatric population group and may assist in implementing effective preventative measures.

\section{Methods}

A retrospective descriptive case audit was conducted at the Pretoria Medico-Legal Laboratory (PMLL) spanning a 10 year period (January 2002 through December 2011) and reviewing records of cases admitted as probable/possible drowning deaths, including all cases which presented as (deceased) bodies retrieved from water, cases where resuscitation had been applied/attempted at the site of immersion and cases where a person who suffered immersion had been admitted to hospital, but did not survive. Demographic details, location of drowning/immersion and selected medico-legal autopsy findings were recorded. Pretoria is the capital city of South Africa and is a large modern city with a population of approximately 2 million people. The study was approved by the Research and Ethics Committee of the Faculty of Health Sciences of the University of Pretoria.

\section{Results:}

A total of 346 cases were identified for inclusion in this study, including all cases which initially presented to us as possible or probable deaths due to drowning. Overall, the total number of bodies admitted to the mortuary over the study period was 23050 . The study cases constituted between 1.1 and $1.8 \%$ of the annual case load at the PMLL. Upon conclusion of the medico-legal autopsy and investigation, 278 of the 346 cases were considered to have died due to drowning, thus collectively comprising $1.2 \%$ of the case load. The drowning cases contributed between $0.8 \%$ to $1.6 \%$ to the total case load. Figure 1 displays the number of drowning cases per year and shows ain relation to the total case load. The trend shows a sharp decline towards 2006, a slight increase and followed by a plateau.

\section{External cause / circumstance of death $(n=346)$ :}

In $20(6 \%)$ of the 346 cases a specific cause of death other than drowning was identified, most of these cases being related to traumatic injury. In $48(14 \%)$ cases no specific or probable cause of death could be established at autopsy. In $80 \%$ (278) of cases the final cause of death was deemed to have been due to (or consistent with) drowning. (Figure 1). Decomposition was present in 45 (13\%) of all study cases. Of the 48 cases where no cause of death could be established, 34 (71\%) showed signs of decomposition and of the 278 cases where drowning was deemed to be the cause of death, 11 cases (4\%) showed signs of decomposition. 


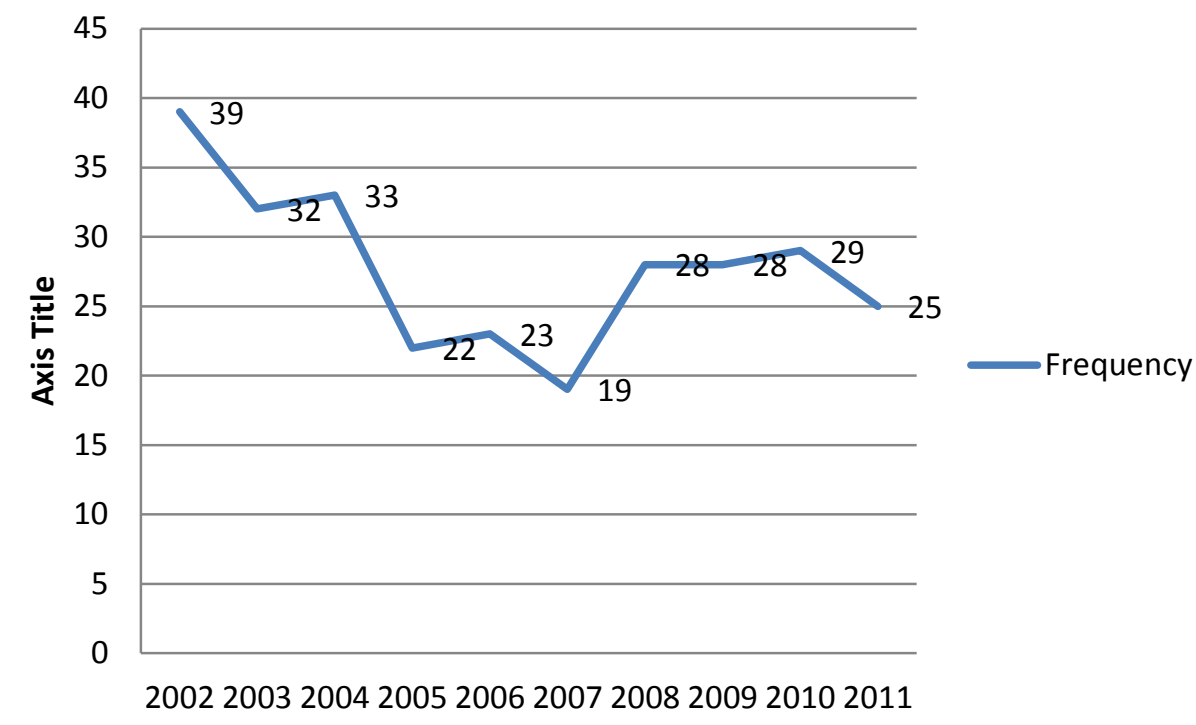

Figure 1: Trend in the number of drownings over the study period.

Deaths due to drowning $(\mathrm{n}=278)$

Demographic details

There were 59 (21\%) females and 219 (79\%) males. Although racial profiling may be problematic and contentious, 197 (71\%) of cases were deemed to be Black, 68 (24\%) were White, $10(4 \%)$ were Colored and 3 $(1 \%)$ were deemed to be Asian. Overall, children accounted for $152(55 \%)$ of cases. More specifically, infants (younger than 1 year of age) comprised 41 (15\%) of the cases, toddlers (aged 1-2 years) constituted $52(19 \%)$ of cases, young children (aged 2-13 years) numbered 49 (18\%) and adolescents (aged 13-18 years) numbered 10 (3\%). Adults (above 18 years) accounted for 126 (45\%) of the cases. Overall, ages ranged from less than one year to 85 years with a mean age of 16.3 years. In the adult population (above 18 years), the mean age was 34.5 years. The demographic details are depicted in Table I.

Table I: Demographic details $(\mathbf{n}=\mathbf{2 7 8})$

\begin{tabular}{|c|c|c|c|c|}
\hline \multicolumn{4}{|c|}{ Gender } & \\
\hline \multicolumn{2}{|c|}{ Male } & \multicolumn{2}{|c|}{ Female } & \\
\hline \multicolumn{2}{|c|}{$219(79 \%)$} & \multicolumn{2}{|c|}{$59(21 \%)$} & \\
\hline \multicolumn{4}{|c|}{ Racial profile } & \\
\hline Black & White & Colored & Asian & \\
\hline $197(71 \%)$ & $68(24 \%)$ & $10(4 \%)$ & $3(1 \%)$ & \\
\hline \multicolumn{5}{|c|}{ Age } \\
\hline $\begin{array}{c}\text { Infants }(<1 \\
\text { year })\end{array}$ & $\begin{array}{l}\text { Toddlers (1-2 } \\
\text { years) }\end{array}$ & $\begin{array}{c}\text { Young children } \\
\text { (2-13 years) }\end{array}$ & $\begin{array}{l}\text { Adolescents } \\
\text { (13-18 years) }\end{array}$ & $\begin{array}{c}\text { Adults }(>18 \\
\text { years) }\end{array}$ \\
\hline $41(15 \%)$ & $52(19 \%)$ & $49(18 \%)$ & $10(3 \%)$ & $126(45 \%)$ \\
\hline
\end{tabular}

Seasonal / month-on-month incidence

On a month to month basis, most drownings occurred in December [48 (17\%)], followed by January and February. (Figure 2). These are our summer months, which collectively accounted for $111(40 \%)$ of all drownings, whilst spring (September - November) yielded 68 (24\%) cases, autumn (March - May) had 53 (19\%) and winter (June - August) 46 (17\%) cases. 


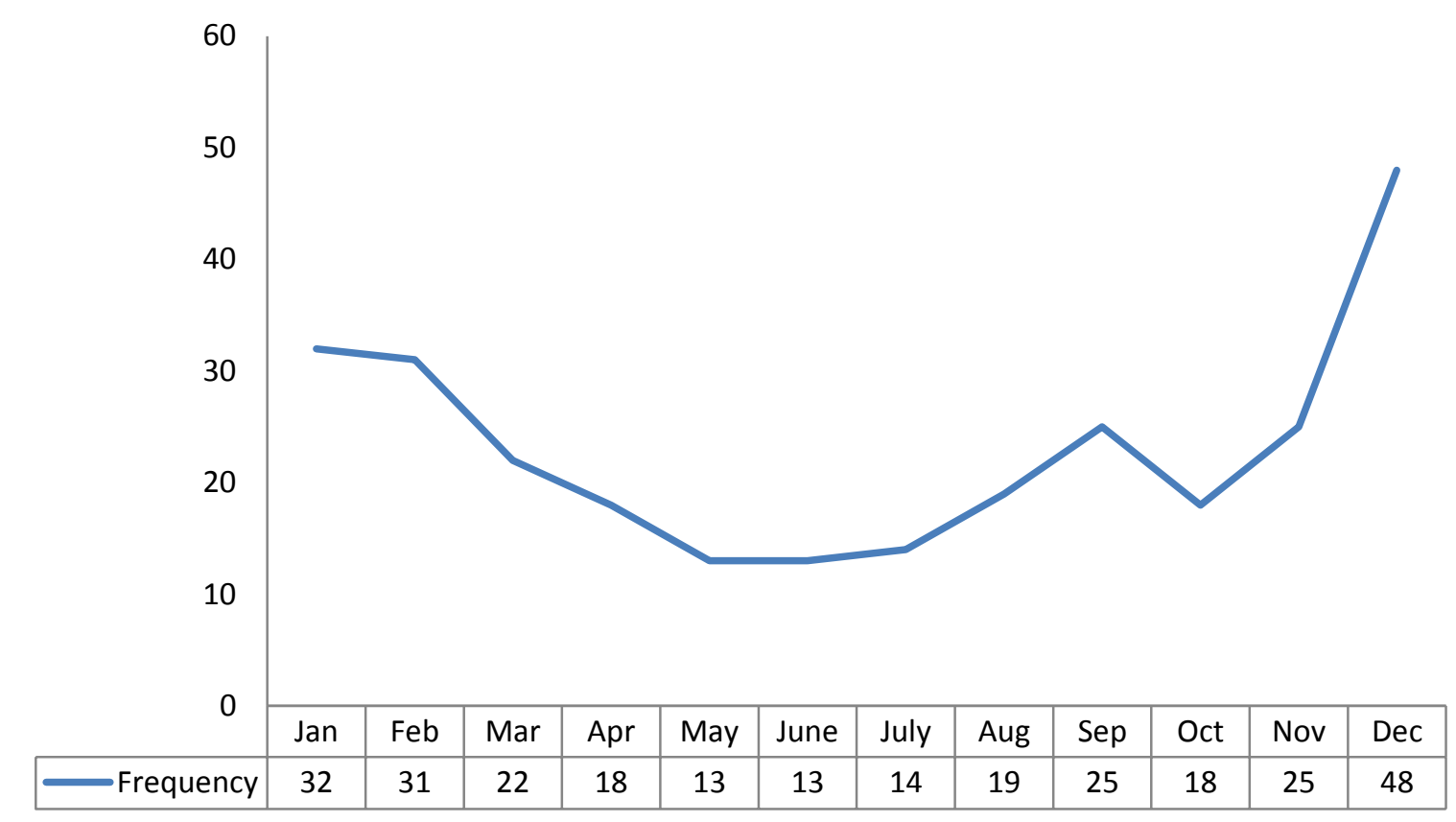

Figure 2: Month-on-month incidence of drowning

Site / Location of drowning

The majority of the drownings occurred in swimming pools [125 cases (38\%)], rivers [43 cases (21\%)], dams [52 cases $(21 \%)]$ and bath tubs [16 cases (5\%)]. (Figure 3)

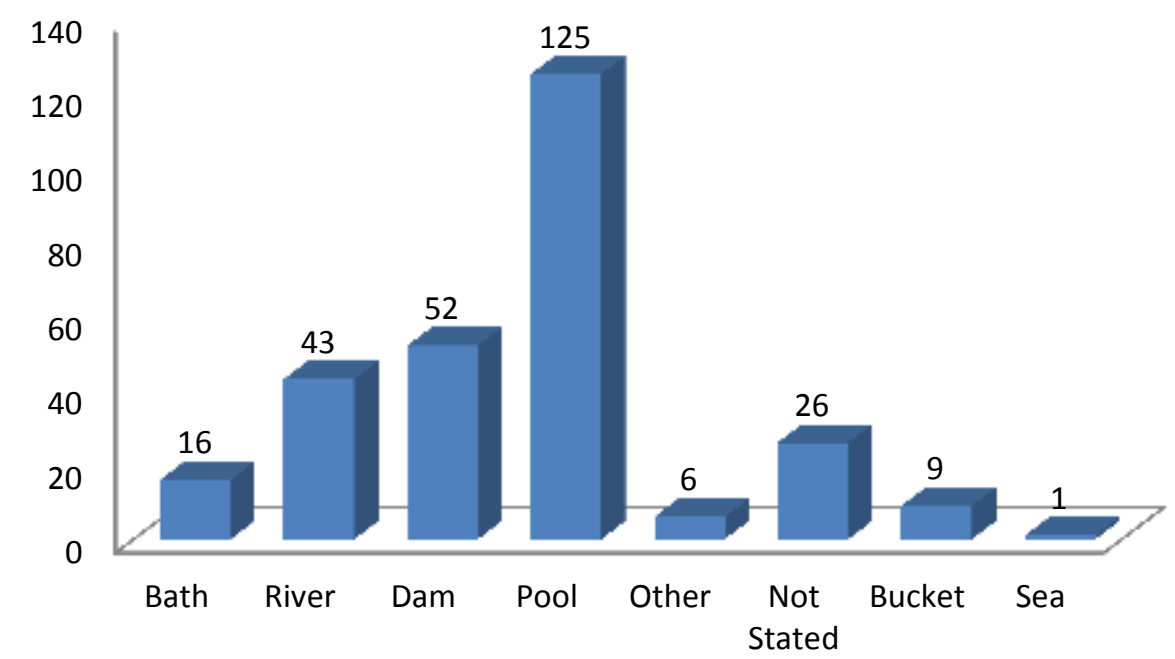

Figure 3: Location of drowning.

The place of drowning was correlated with the age groups, specifically in respect of infants, toddlers and adults. Overall, 85 (56\%) of child drownings took place in swimming pools, including $23(56 \%)$ of the infant drownings and $36(69 \%)$ of toddler drownings. There were $7(16 \%)$ infant drownings in buckets. Adult drownings occurred mostly in swimming pools [40 cases (32\%)], rivers [35 cases (28\%)] and dams [29 cases $(23 \%)]$. One person was transferred from the coast to Pretoria for treatment, after immersion in the sea. Eleven adults drowned in a bath: 6 were known to be epileptics, 2 had underlying cardiac pathology and in 1 prescription medication may have played a predisposing or contributory role in the drowning. (Table II) 
Table II: Location of drowning in relation to age group

\begin{tabular}{|c|c|c|c|c|c|c|}
\hline $\begin{array}{l}\text { Drowning } \\
\text { site/setting: }\end{array}$ & Infant & Toddler & Child & Adolescent & Adult & Total: \\
\hline Bathtub & $1(2 \%)$ & $2(4 \%)$ & $2(4 \%)$ & $0(\%)$ & $11(9 \%)$ & $16(6 \%)$ \\
\hline River & $1(2 \%)$ & $3(6 \%)$ & $3(6 \%)$ & $1(10 \%)$ & $35(28 \%)$ & $43(15 \%)$ \\
\hline Dam & $0(\%)$ & $2(4 \%)$ & $17(35 \%)$ & $4(40 \%)$ & $29(23 \%)$ & $52(19 \%)$ \\
\hline Pool & $23(56 \%)$ & $36(69 \%)$ & $22(45 \%)$ & $4(40 \%)$ & $40(32 \%)$ & $125(45 \%)$ \\
\hline Other & $1(2 \%)$ & $2(4 \%)$ & $0(\%)$ & $0(\%)$ & $3(2 \%)$ & $6(2 \%)$ \\
\hline Not stated & $8(20 \%)$ & $6(12 \%)$ & $5(10 \%)$ & $1(10 \%)$ & $6(5 \%)$ & $26(9 \%)$ \\
\hline Bucket of water & $7(17 \%)$ & $1(2 \%)$ & $0(\%)$ & $0(\%)$ & $1(1 \%)$ & $9(3 \%)$ \\
\hline Sea & $0(\%)$ & $0(\%)$ & $0(\%)$ & $0(\%)$ & $1(1 \%)$ & $1(0.4 \%)$ \\
\hline TOTAL & 41 & 52 & 49 & 10 & 126 & 278 \\
\hline
\end{tabular}

Features of resuscitation / medical intervention

In the majority of cases the decedents had died at the scene with no resuscitative measures having been applied [169 cases (61\%)]. In 109 cases (39\%) efforts at resuscitation were initiated. Of the latter group $49(45 \%)$ patients were however declared as being dead on arrival at the hospital emergency room. Of the patients admitted to hospital, the duration of admission ranged from minutes/hours to 45 days.

\section{Post mortem anatomical / pathological findings}

External signs indicative of immersion included maceration of the skin [9 cases (2\%)], washerwoman hands / feet $[28$ cases $(10 \%)]$ and cutis anserina [19 cases (7\%)]. A plume of froth at the nostrils was recorded in 85 cases $(31 \%)$. The specific term/entity of emphysema aquosum was used / recorded in the autopsy reports in 92 cases $(33 \%)$ and hemorrhages in the middle ear and mastoid were reported in 14 cases $(5 \%)$. Intimal staining of the aorta was reported in 42 of the cases $(15 \%)$. Histological examination was undertaken in $117(42 \%)$ of drowning cases. In only 15 of the 126 adults (12\%), features of an underlying medical disease were present at autopsy. There were 9 cases (3.2\%) of epilepsy (this include 6 drownings in the bathtub) and 6 cases (2.2\%) of cardiac abnormalities including ischemic heart disease.

\section{Blood alcohol analysis}

Post mortem blood alcohol analysis was undertaken in $113(41 \%)$ of the 278 drowning cases and alcohol was detected in $48(42 \%)$ of these samples - with 40 cases $(35 \%)$ having blood alcohol concentrations in excess of $0.05 \mathrm{~g}$ per $100 \mathrm{ml}$. The blood alcohol levels ranged from $0.05-0.43 \mathrm{~g}$ per $100 \mathrm{ml}$. Only three of the 48 positive results came from female victims. No alcohol was detected in any of the adolescent victims for whom blood was tested.

\section{Discussion:}

Our study showed that immersion related deaths and bodies retrieved from water constitute nearly $2 \%$ of the total annual case load admitted to the medico-legal mortuary in the capital city of South Africa. The cases in which drowning was eventually deemed to be the cause of death accounted on average for $1.2 \%$ of the annual case load at our mortuary. This is very similar to that reported by the San Diego Medical Examiner for 2011, where deaths from drowning constituted 38 (1.3\%) of 2853 cases which were autopsied. ${ }^{19}$ This figure is however significantly lower than that reported by Shetty et al from India (1994-2005), where drowning deaths accounted for $13 \%$ of the forensic mortuary case load. ${ }^{20}$ The majority of the decedents admitted to the Pretoria MLL had been declared dead at the scene $(61 \%)$. This is a substantially lower figure than that which was reported by Fang et al (China 2001-2005), where 86.6\% of drowning related deaths had been declared dead at the scene and before transfer to a medical facility could take place. ${ }^{16}$ 
Drowning deaths in children is a preventable cause of death and is of universal concern. ${ }^{4}$ In our study $55 \%$ of all drowning deaths involved children under the age of 18. Another sad but striking statistic from our study is that infants under the age of 1 year comprised $15 \%$ of drowning cases (the majority of whom had died in swimming pools and buckets). Most childhood drownings however, involved toddlers and young children under the age of 13 years, who drowned in swimming pools. Bamber et al (London 1995-2010) recorded 28 cases of drowning in an urban setting over a 16 year period, involving children between the ages of 7 days and 16 years. ${ }^{15}$ Fang et al reported on drowning deaths in China in children aged from 1 to 14 years, for the period 2001-2005. ${ }^{16}$ The majority of these deaths involved older children (more commonly in the age groups 5-9 and then 10-14 years) and occurred in natural bodies of water such as wells and ponds, with only $3 \%$ of drownings occurring at home. ${ }^{16}$ Pretoria has a hot subtropical climate and has many affluent neighbourhoods where most houses have private swimming pools. This may in part explain the relatively high incidence of drownings amongst children in swimming pools in Pretoria. Lack of adequate supervision and ineffective physical barriers to prevent free access to pools probably also play a major role here. Amongst underprivileged groups, leisure swimming activities tend to take place in open bodies of water, typically in the summer months, where conditions may be very dangerous and where such activity is often unsupervised. The findings of our study suggest that urgent measures are required to help prevent child drownings in private swimming pools, including especially mandatory physical barriers / safety nets. As stated by Richards however, public education plays an important role in preventing these deaths - including education regarding basic life support (resuscitation) measures, supervision of children and the dangers of alcohol consumption and swimming. ${ }^{3}$

Immersion related deaths are problematic entities for forensic pathologists: the diagnosis of drowning cannot be definitely made based on autopsy findings alone and rests in the first instance on exclusion of other causes of death. In most cases of drowning, it is probably prudent for the pathologist to formulate the cause of death (based on autopsy findings only) as being "consistent with drowning". In particular, putrefactive changes will compromise or preclude the firm diagnosis of drowning. ${ }^{9}$ In our study, $13 \%$ of the bodies retrieved from water showed signs of decomposition. In bodies retrieved from water where no definitive cause of death was ascertained, $71 \%$ of cases showed signs of decomposition, the latter thus probably undermining the (reliable) diagnosis of drowning. In our study, only $4 \%$ of cases which were diagnosed as death due to drowning, had features of decomposition. Ambade et al (1999-2008) reported on 353 bodies retrieved from water in India: $31.2 \%$ of those bodies were decomposed. They reported however that drowning was ascertained as the cause of death in $75.5 \%$ of the decomposed bodies and $95.9 \%$ in non-decomposed bodies. ${ }^{7}$

As there are no pathognomonic features of drowning, the pathologist should record all potentially relevant "nonspecific" features (including the presence of a plume of froth at the nostrils/mouth, a fluid medium in stomach, middle ear haemorrhages, etc.) and then review the entire case before concluding the diagnosis. ${ }^{21}$ In our study, a plume of froth, emphysema aquosum and Niles hemorrhages were the most commonly recorded of these nonspecific anatomical / pathological findings. Tsokos et al reported hemolytic staining of the aorta to be present in approximately $5 \%$ of drowning cases. ${ }^{11}$ Our study indicated this feature to be somewhat more common (15\%). Cobbett et al also emphasized the importance of conducting a full post mortem examination on all cases of drowning as this can help to identify underlying disease processes such as cardiovascular pathology which could have contributed to the death. ${ }^{12}$ Underlying natural pathology was only present in 15 of the adult drownings. One reason for this might be the relative young mean age of 34.5 years in the adult population group.

The association between high blood alcohol levels and drownings has been documented, with alcohol consumption being regarded as a risk factor for both injury and deaths due to immersion. ${ }^{3}$ Binge drinking is common in South Africa and has been reported to be associated with a higher risk for drowning amongst adult men. ${ }^{18}$ For the period 2001-2005 Donson et al reported that in drowning deaths in 5 large cities in South Africa, alcohol was detected in $40 \%$ of cases, with one third of these deceased individuals having considered to be "drunk to very drunk". ${ }^{18}$ Our study found that 2 out of five (adult) victims of drowning had consumed alcohol and that in $35 \%$ of these positive cases had a BAC in excess of $0.05 \mathrm{~g} / 100 \mathrm{ml}$. Cobbett et al (Auckland - 20092011) found alcohol intoxication in $17.6 \%$ of cases (all of which were male). ${ }^{12}$

\section{Conclusions:}

In Pretoria drowning deaths are most common amongst children who die in swimming pools, with infants under one year representing an inordinately high percentage of cases. These should all be deemed preventable deaths and renewed efforts should be made at improving public awareness and implementing preventive measures. Amongst adults, alcohol is again seen to be present in the blood of many victims of drowning. More should be done to raise awareness of the risk of alcohol consumption in conjunction with swimming activities. The diagnosis of drowning is not easily or reliably arrived at on the basis of autopsy investigation alone and thorough investigation of immersion-related deaths should be done, to ensure a better understanding of the 
prevalence and circumstances of drownings in our society. A more detailed examination, including full histological examinations could in a proportion of the young adults provide an underlying disease process not identified with macroscopic dissection.

\section{References:}

1. van Beeck EF, Branche CM, Szpilman D, Modell JH, Bierens JJ. A new definition of drowning: towards documentation of a global public health problem. Bull World Health Organ. 2005;83:853-6.

2. Roll HF. Leerboek der Gerechtelijke Geneeskunde voor de scholen tot opleiding van Ind. Artsen, 'sGravenhage, Martinus Mijhoff, 1918.

3. Richards DB. Drowning. La noyade. African Journal of Emergency Medicine. 2011;1:33-38.

4. M. Linnan, A. Rahman, J. Scarr, T. Reinten-Reynolds, H. Linnan, J. Rui-wei, S. Mashreky, S. Shafinaz, S. Bose, E. Finkelstein, F. Rahman. Child Drowning: Evidence for a newly recognized cause of child mortality in low and middle income countries in Asia. Special Series on Child Injury 2012. Available from: http://www.unicef.or.jp/library/pdf/Child_Drowning_FINAL_Office_of_Research.pdf Accessed on 3 October 2014.

5. http://www.who.int/mediacentre/factsheets/fs347/en/. Accessed on 3 October 2014.

6. Byard RW. Immersion deaths and drowning: issues arising in the investigation of bodies retrieved from water. Forensic Sci Med Pathol. 2014 April 22 (Epub ahead of print)

7. Ambade VN, Kukde HG, Malani A et al. Decomposed and non-decomposed bodies retrieved from water: a comparative approach. Med Sci Law 2013;53:12-18

8. Saukko P, Knight B. Knights Forensic Pathology. $3^{\text {rd }}$ Edition. Arnold publishers 2004. p395-411.

9. Piette MHA, de Letter EA. Drowning: Still a difficult autopsy diagnosis. Forensic Sci Int 2006;163:19.

10. Papadodima SA, Athanaselis SA, Skliros E, Spiliopoulou CA. Forensic investigation of submersion deaths. Int J Clin Pract 2010;64(1):75-83.

11. Tsokos M, Cains G, Byard RW. Hemolytic staining of the intima of the aortic root in fresh water drowning - a retrospective study. Am J Forensic Med Pathol. 2008;29:128-130.

12. Cobbett H, Morrow P, Stables SR. Sink or swim: The advantages of full postmortem examination in cases of drowning. Acad Forensic Pathol 2014;4(2):214-219.

13. Driscoll TR, Harisson JA, Steenkamp M. Review of the role of alcohol in drowning associated with recreational aquatic activity. Inj Prev 2004;10:107-113.

14. Warner M, Smith GS, Langley JD. Drowning and alcohol in New Zealand: what do the coroner's files tell us? Aust N Z J Public Health 2000;24:387-90.

15. Bamber AR, Pryce JW, Ashworth MT, Sebire NJ. Immersion-related deaths in infants and children: autopsy experience from a specialist center. Forensic Sci Med Pathol 2014 June 4 [Epub ahead of print]

16. Fang Y, dai L, Jaung MS, Chen Xm Yu S, Xiang H. Child drownings in Xiamen city and suburbs, People's Republic of China, 2001-5. Inj Prev 2007;13:339-343

17. Mortality and causes of death in SouthAfrica, 2010: Findings from death Notification. Available from http://www.statssa.gov.za/publications/p03093/p030932010.pdf Accessed 3 October 2014.

18. Donson H, van Niekerk A. Unintentional drowning in urban South Africa: a retrospective investigation, 2001-2005. Int J Inj Contr Saf Promot 2013;20(3):218-226.

19. Wagner G. San Diego County Department of the Medical Examiner 2011 Annual report. Available from http://www.sandiegocounty.gov/me/docs/SDME_Annual_Report 2011.pdf Accessed 3 October 2014

20. Shetty BSK, Shetty MS. Epidemiology of drowning in Mangalore, a coastal Taluk of South India. J Forensic Leg Med. 2007 Oct;14(7):410-5.

21. Byard RW. Aortic intimal staining in drowning. Forens Sci Med Pathol 2014 Apr 22. [Epub ahead of print] 